\title{
L'huile de colza : une huile multi-usages en devenir
}

Pierre-Yves VIGNERON

Joel CAIGNIEZ

Bernard STOCLIN

Jean-Pierre BREGNARD

LESIEUR, 101 route de Bourbourg,

BP 89,

59412 Coudekerque-Branche Cedex, France <bstoclin@lesieur.fr>
Article reçu le 23 décembre 2005 accepté le 12 novembre 2006

\begin{abstract}
Ageing tests have been carried out with refined rapeseed oil in different conditions (transparent and non transparent bottles, moderate and dim natural light exposure, room and refrigerator temperature).

The results mainly based on sensory evaluation show that rapeseed oil keeps a rather good quality if kept in a non transparent bottle and best if stored in the refrigerator. Results of frying tests under household conditions show that chemical degradation of rapeseed oil is lower than for sunflower oil. But for both oils, the level of degradation compounds are below the $25 \%$ polar compounds limit, assuming that the consumer does respect the recommendations on the label i.e. temperature $\leq 180^{\circ} \mathrm{C}$ and not more than 10 fryings. Although the French regulation does not allow until now to use oils for frying purpose with more than $2 \%$ of linolenic acid of their fatty acid composition, results demonstrate that rapeseed oil could be used worthwhile for frying. However on the basis of room odor tests, rapeseed oil might not be accepted by some consumers due to its fishy odor during frying operation. Therefore from a marketing point of view, it is advisable to limit the use of rapeseed oil to seasoning and cooking purposes.
\end{abstract}

Key words: rapeseed oil, sensory evaluation, ageing test, frying degradation

L'intérêt nutritionnel de l'huile de colza est désormais reconnu par les nutritionnistes.

Sa consommation permet de combler en partie le déficit en acide linolénique souligné dans diverses études [1-3].

Sa composition en acides linoléique et linolénique, et le taux de répartition de ce dernier en position sn2 sont autant d'arguments en sa faveur, car ils permettent à l'huile de colza de se rapprocher des recommandations en matière d'apports nutritionnels [4].

Cependant, l'huile de colza comme l'huile de soja a été souvent considérée à tort ou à raison comme une huile fragile, particulièrement à chaud, ce qui a conduit la législation française à limiter aux usages d'assaisonnement les huiles dont la teneur en acide linolénique est supérieure à $2 \%$. De plus, les teneurs en acide linolénique du colza (en moyenne $8 \%$ ) et en triglycérides comportant au moins deux acides linolénique $(\approx 3,5 \%)$ pourraient être à l'origine de son instabilité à la lumière qui se traduit par l'apparition de goûts déplaisants (rance, poisson) au cours du temps.

En dépit d'un raffinage soigné, en particulier éliminant toutes traces de pigments chlorophylliens avant la désodorisation, certaines huiles de colza ont encore après désodorisation un fruité net s'apparentant à un goût de graines vertes ou de haricot. Notre expérience a montré que ces huiles pouvaient dans certains cas prendre rapidement un goût déplaisant pour le consommateur. Notre étude a comporté deux parties : I'une consiste à évaluer les performances de l'huile de colza en usages à température ambiante, l'autre aux chauffages.

\section{Usages à température ambiante}

Le consommateur attend que l'huile raffinée soit sans goût particulier tout au long de la durée d'utilisation conseillée.

Nos essais ont eu pour but d'évaluer la stabilité organoleptique de l'huile de colza dans des conditions de conservation proches de celles que l'on pourrait trouver chez le consommateur.

Une huile commercialisée de colza de pression raffinée a alors été soumise à deux modes de vieillissement.

Le premier mode est un vieillissement naturel à température ambiante effectué soit dans une pièce exposée à l'ouest, soit au nord. Dans le premier cas, la lumière est diffuse, modérément intense, correspondant à une exposition d'au moins 300 lux, pouvant aller jusqu'à 1000 lux. Dans le second cas, la lumière est diffuse et atténuée, correspondant à une exposition d'au moins 50 lux, pouvant aller jusqu'à 100 lux.

Pour chaque emballage testé, 12 bouteilles remplies sont placées sur une étagère pendant 12 semaines.

Chaque semaine, une bouteille est retirée puis conservée au congélateur pour être ultérieurement analysée.

Le second mode de vieillissement est un test qui se rapproche des conditions d'utilisation par le consommateur. Ce test dit test d'usage consiste à soutirer de la bouteille de 1 litre, 2 cuillères à soupe soit $\approx 20 \mathrm{~g}, 3$ fois par semaine, le flacon étant exposé à la lumière du jour diffuse soit dans une pièce exposée à l'ouest, soit au nord. 
Trois séries de bouteilles sont ainsi préparées, la première pour les mesures et tests oranoleptiques après un mois de stockage, les deux autres après deux et trois mois, chacune de ces bouteilles contenant à la fin du test respectivement environ les trois quarts, la moitié et le quart de leur volume initial en huile.

Ce protocole a aussi été effectué avec des bouteilles conservées au réfrigérateur.

Dans le cas du test d'usage au réfrigérateur, la bouteille d'huile était sortie dans la matinée et laissée à température ambiante pendant 2 h 30 , temps estimé de préparation et de prise des repas, les prélèvements étaient alors faits puis la bouteille était remise au réfrigérateur.

Pour les deux modes de vieillissement, la même huile est contenue dans deux types d'emballage en PET, I'un transparent, l'autre coloré et opaque. L'éventuelle détérioration des échantillons est évaluée par l'analyse sensorielle et quelques analyses physico-chimiques.

Les tests organoleptiques sont réalisés par un panel de 6 à 8 personnes entraînées dans une salle dédiée, équipée de six boxes isolés éclairés par une lumière rouge ne permettant pas de différencier les échantillons par leur couleur.

Les huiles sont préchauffées à $36 \pm 2{ }^{\circ} \mathrm{C}$ pendant au moins 30 minutes avant d'être évaluées par les panélistes. Le jury a au maximum six échantillons à évaluer, le test ne se faisant que dans la matinée.

Chaque box est équipé $d^{\prime}$ un bloc maintenant les coupelles d'huile à $\approx 36^{\circ} \mathrm{C}$ et d'un écran digital sur lequel apparaît la feuille de dégustation. Les descripteurs y figurant sont : fruité, goût de verdure ou de haricot, plastique, rance, poisson, brûlé et goût non identifié.

Avec la souris, le panéliste positionne sur une ligne non graduée correspondant au défaut perçu le curseur en fonction de l'intensité ressentie (faible à l'extrémité gauche, forte à droite) puis donne une note d'appréciation globale entre 0 et 10. L'ensemble de ces éléments est traité par le logiciel informatique FIZZ (Biosystems - Couternon 21560 France).

L'huile est jugée organoleptiquement acceptable si la moyenne des notes d'appréciation globale est supérieure ou égale à 7,0 .

En complément à l'évaluation sensorielle, on évalue le degré d'oxydation à l'aide des méthodes habituelles normalisées ISO à savoir l'indice de peroxyde (NF EN ISO 3960), I'indice de para-anisidine (NF EN ISO 6885) et les absorptions dans I'ultraviolet (NF EN ISO 3656).

La détermination de la teneur cumulée en composés volatils et de certains composés spécifiques est effectuée par la technique de l'espace de tête dynamique et chromatographie en phase gazeuse sur colonne apolaire BP1 [5].

\section{Résultats : vieillissement naturel}

Notre expérience sur plusieurs années a établi que dans les conditions de ce test une huile riche en acide linolénique n'est organoleptiquement acceptable qu'au maximum 3-4 semaines en emballage transparent lorsqu'elle est exposée à la lumière naturelle modérément intense (exposition ouest de 300 à 1000 lux environ) et est à la limite de l'acceptabilité à partir de 6 à 8 semaines à la lumière naturelle atténuée (exposition nord de 50 à 100 lux environ). Les résultats du tableau 1 confirment cette tendance et montrent que l'emballage opaque permet de garantir le goût quelles que soient les conditions d'exposition. L'évolution de l'indice de peroxyde est sensiblement ma même que celle observée avec l'emballage transparent exposé à une lumière diffuse faible.

\section{Résultats : test d'usage}

Ce test est plus sévère que le précédent puisque l'espace de tête de la bouteille est renouvelé à chaque prélèvement. Il paraît cependant mieux anticiper ce que pourraient être l'usage et éventuellement les réclamations du consommateur.

Les résultats montrent que I'huile de colza est sensiblement plus stable au réfrigérateur qu'à température ambiante, que la bouteille soit opaque ou transparente (tableau 2).

Exposé à la lumière naturelle modérément intense et à température ambiante, le produit est fragilisé d'autant plus rapidement qu'il est en bouteille transparente et est à la limite de l'acceptabilité même en emballage opaque.

Au fur et à mesure de la diminution de la note d'appréciation sensorielle globale, on constate une nette augmentation de l'indice de peroxyde et de l'extinction spécifique à $232 \mathrm{~nm}$. Par contre, quels que soient le mode d'exposition et l'emballage examiné, l'extinction à $270 \mathrm{~nm}$ n'évolue pas au cours du test tandis que l'indice de para-anisidine n'augmente significativement qu'au troisième mois. Le dosage des composés volatils totaux du pentane et du c, t et $t, t$ décadiénal confirme que la dégradation de l'huile dans des conditions d'usage " consommateur » est sensiblement ralentie quand l'huile est remise au réfrigérateur après chaque ouverture.

La qualité de l'huile au départ est importante car comme le montre le tableau 3, une huile de qualité inférieure (note 7,2 ) sera rapidement inacceptable d'un point de vue organoleptique dans les conditions de ce test d'usage.

\section{Usages à chaud}

Des essais de chauffages et de fritures ont été réalisés avec une friteuse électrique à zone froide de marque Roller Grill (La Redoute - Roubaix France 59100) dans les conditions suivantes.

\section{Test de chauffages}

$2,8 \mathrm{~kg}$ d'huile.

Chauffage vers $190^{\circ} \mathrm{C} \approx 6 \mathrm{~min}$.

Palier de 20 min à température.

Tableau 1. Vieillissement naturel.

\begin{tabular}{|c|c|c|c|c|c|c|c|c|c|c|c|c|}
\hline & & \multicolumn{11}{|c|}{ Temps (semaines) } \\
\hline & & \multirow[t]{2}{*}{0} & \multicolumn{2}{|c|}{3} & \multicolumn{2}{|c|}{4} & \multicolumn{2}{|c|}{5} & \multicolumn{2}{|c|}{6} & \multicolumn{2}{|c|}{8} \\
\hline & & & $A^{*}$ & $B^{* *}$ & A & B & A & B & A & B & A & B \\
\hline \multirow{2}{*}{$\begin{array}{l}\text { Bouteille } \\
\text { transparente }\end{array}$} & Note sensorielle moyenne & 7,85 & & 7,25 & 7,45 & 6,85 & 7,4 & 6,5 & 7,25 & 6,4 & 7 & l \\
\hline & Indice de peroxyde & 0,3 & 0,6 & 1,3 & 0,7 & 1,5 & 0,7 & 1,9 & 0,8 & 2,5 & 1 & \\
\hline \multirow{2}{*}{$\begin{array}{l}\text { Bouteille colorée } \\
\text { opaque }\end{array}$} & Note & 7,85 & & & 7,65 & 7,3 & I & 7,4 & 7,4 & 7,2 & 7,35 & 7,1 \\
\hline & Indice de peroxyde & 0,3 & & & 0,4 & 0,5 & & & 0,4 & 0,6 & 0.5 & 0.8 \\
\hline
\end{tabular}

${ }^{*} \mathrm{~A}$ : Lumière naturelle atténuée ; ${ }^{* * B}$ : Lumière naturelle modérément intense. 


\begin{tabular}{|c|c|c|c|c|c|c|c|c|c|c|c|}
\hline & & \multicolumn{10}{|c|}{ Durée } \\
\hline & & \multirow[t]{2}{*}{0 mois } & \multicolumn{3}{|c|}{1 mois } & \multicolumn{3}{|c|}{2 mois } & \multicolumn{3}{|c|}{3 mois } \\
\hline & & & $4^{\circ} \mathrm{C}$ & $A^{*}$ & $\mathbf{B}^{* *}$ & $4{ }^{\circ} \mathrm{C}$ & A & B & $4^{\circ} \mathrm{C}$ & A & B \\
\hline \multirow{8}{*}{$\begin{array}{l}\text { Bouteille } \\
\text { transparente }\end{array}$} & Note sensorielle moyenne & 7,85 & 7,6 & 7,2 & 6,3 & 7,2 & 6,7 & & 7,4 & 7,1 & \\
\hline & Indice de peroxyde (meq/kg) & 0,6 & 0,8 & 1,0 & 6,1 & 1,1 & 6,3 & & 2,7 & 15 & \\
\hline & Indice de para-anisidine & 2,1 & 2,3 & 2,2 & & 2,4 & 3,1 & & 2,2 & 3,3 & \\
\hline & Extinctions spécifiques E232 & 3,9 & 3,9 & 3,9 & & 3,9 & 5,6 & & 3,8 & 7,1 & \\
\hline & E270 & 0,6 & 0,6 & 0,6 & & 0,6 & 0,6 & & 0,6 & 0,7 & \\
\hline & Volatils totaux (mg/kg) & 36 & 38 & 80 & 150 & 135 & 539 & & 190 & 699 & \\
\hline & Pentane (mg/kg) & 7 & 7 & 12 & 38 & 28 & 143 & & 50 & 195 & \\
\hline & $\mathrm{c}, \mathrm{t}$ + tt décadiénal $(\mathrm{mg} / \mathrm{kg})$ & 5 & 5 & 13 & 32 & 36 & 206 & & 68 & 280 & \\
\hline \multirow{8}{*}{$\begin{array}{l}\text { Bouteille } \\
\text { colorée } \\
\text { opaque }\end{array}$} & Note sensorielle moyenne & 7,85 & 8,0 & & 7,0 & 7,6 & & 6,8 & 7,4 & & 6,9 \\
\hline & Indice de peroxyde (meq/kg) & 0,6 & 0,5 & & 1,6 & 0,9 & & 6,5 & 0,9 & & 19,6 \\
\hline & Indice de para-anisidine & 2,1 & 2,3 & & 2,4 & 2,4 & & 2,6 & 2,2 & & 4,1 \\
\hline & Extinctions spécifiques E232 & 3,9 & 3,8 & & 4,2 & 3,8 & & 5,8 & 4,0 & & 7,5 \\
\hline & E270 & 0,6 & 0,6 & & 0,6 & 0,6 & & 0,7 & 0,6 & & 0,7 \\
\hline & Volatils totaux $(\mathrm{mg} / \mathrm{kg})$ & 36 & & & 64 & 164 & & 370 & 270 & & 782 \\
\hline & Pentane $(\mathrm{mg} / \mathrm{kg})$ & 7 & & & 12 & 46 & & 107 & 64 & & 224 \\
\hline & $\mathrm{c}, \mathrm{t}+\mathrm{tt}$ décadiénal $(\mathrm{mg} / \mathrm{kg})$ & 5 & & & 11 & 62 & & 157 & 92 & & 314 \\
\hline
\end{tabular}

${ }^{*} A$ : Lumière naturelle atténuée ; ${ }^{* *} B$ : Lumière naturelle modérément intense.

Tableau 3. Test d'usage.

\begin{tabular}{|c|c|c|c|c|c|}
\hline & & \multicolumn{4}{|c|}{ Durée } \\
\hline & & \multirow[t]{2}{*}{0 mois } & \multicolumn{3}{|c|}{1 mois } \\
\hline & & & $4^{\circ} \mathrm{C}$ & $\mathbf{A}^{*}$ & $\mathbf{B}^{* *}$ \\
\hline \multirow{5}{*}{$\begin{array}{l}\text { Bouteille } \\
\text { transparente }\end{array}$} & Note & 7,2 & 6,7 & 6,3 & \\
\hline & Indice de peroxyde (meq/kg) & 0,6 & 1,1 & 3,4 & \\
\hline & Indice de para-anisidine & 1,3 & & 1,9 & \\
\hline & Extinctions spécifiques E232 & 3,8 & 3,7 & 3,9 & \\
\hline & $E 270$ & 0,6 & 0,6 & 0,6 & \\
\hline \multirow{5}{*}{ Bouteille colorée } & Note & 7,2 & 7,4 & & 6,5 \\
\hline & Indice de peroxyde (meq/kg) & 0,6 & 0,6 & & 3,0 \\
\hline & Indice de para-anisidine & 1,3 & & & 1,3 \\
\hline & Extinctions spécifiques E232 & 3,8 & 3,8 & & 3,9 \\
\hline & E270 & 0,6 & 0,6 & & 0,6 \\
\hline
\end{tabular}

${ }^{*} A$ : Lumière naturelle atténuée ; ${ }^{* *} B$ : Lumière naturelle modérément intense.

\section{Test de fritures}

$2,8 \mathrm{~kg}$ d'huile.

Chauffage vers $190^{\circ} \mathrm{C} \approx 6 \mathrm{~min}$.

Palier de 10 min à température.

$400 \mathrm{~g}$ de frites lavées essorées, pochage $4 \mathrm{~min}$.

Laisser revenir à température $\approx 1 \mathrm{~min}$ puis dorage $1 \mathrm{~min}$.

Égouttage : 14 minutes.

Trois chauffages ou fritures étaient réalisées quotidiennement, la température revenant à $\approx 45^{\circ} \mathrm{C}$ avant le chauffage suivant.

Les variations de régulation de ce type de matériel commercial sont importantes (180 à $195^{\circ} \mathrm{C}$ ).

Des échantillons d'environ $40 \mathrm{~g}$ sont prélevés de cinq en cinq chauffages ou fritures afin de réaliser les déterminations suivantes :

- Composés polaires

Chromatographie liquide haute performance avec détection par diffusion de lumière (ELSD500 Alltech Templemars 59175 France) [5].
- Glycérides polymérisés

Chromatographie liquide d'exclusion à haute performance (NF EN ISO 16931).

- Non-chromatographié et teneur en acides gras.

La méthode consiste à méthyler le corps gras en présence d'un étalon interne (heptadecanoate de méthyle) qui sert à calculer après chromatographie en phase gazeuse, la teneur de chaque acide gras pour en déterminer la perte éventuelle [6].

- Acidité

\section{NF EN ISO 660.}

\section{- Couleur}

Mesure du pourcentage de lumière transmise à $420 \mathrm{~nm}$ au travers de l'échantillon en cuve de $10 \mathrm{~mm}$ par rapport à l'eau.

- Tocophérols

Chromatographie liquide haute performance et détection par fluorimétrie [5]. 
- Hauteur de mousse

Évaluation en éprouvette selon le protocole décrit en [7].

Préalablement aux essais de chauffage et de friture, on s'est assuré que ni I'huile de colza, ni les friteuses utilisées n'avaient été contaminées par un agent « antimoussant » à l'aide d'un test pratique développé en interne. Ce point est très important car la présence de tels additifs dont l'objectif est de ralentir la dégradation des huiles, fausserait tous les résultats.

\section{Résultats}

Il est difficile de comparer les résultats avec ceux d'autres auteurs qui ont opéré des chauffages en continu $[8,9]$.

Dans les conditions de nos tests, il faut environ 13 chauffages et 20 fritures pour atteindre la limite des $25 \%$ de composés polaires prévue par la législation française (tableau 4).

A ce niveau, les quantités d'acides polyinsaturés diminuent respectivement d'environ $18 \%$ (C18:2 cis) et de $31 \%$ (C18:3 cis) sans qu'il y ait de formation d'acides gras trans. La mousse devient abondante à partir de 20-25\% de composés polaires avec risque de débordement de la friteuse dès que la hauteur est supérieure à $60 \mathrm{~mL}$.

II n'y a quasiment plus de tocophérols à partir du $10^{\mathrm{e}}$ chauffage ou friture. Si l'on compare par rapport à une huile de tournesol soumise aux mêmes conditions de chauffage et de friture (tableau 5), on constate que l'huile de colza se dégrade moins vite que le tournesol. Pour cette dernière, la limite des $25 \%$ de composés polaires est atteinte aux environs des 10 chauffages et de 15 fritures, ce qui confirme les recommandations faites au consommateur.

Des essais de cuisson à la poêle ont été réalisés dans les conditions suivantes avec une poêle de diamètre $22 \mathrm{~cm}$ (marque Téfal SEB -
Selongey 21261 France) chauffée sur une plaque chauffante électrique de $1500 \mathrm{~W}$.

$50 \mathrm{~g} \mathrm{~d}$ 'huile de colza étaient chauffés à $150^{\circ} \mathrm{C}-160^{\circ} \mathrm{C}$ avant d'y ajouter $225 \mathrm{~g}$ de pommes de terre préalablement coupées en petits cubes, lavées puis bien essorées. La cuisson durait 8 minutes.

Un autre essai a été réalisé dans les mêmes conditions pour cuire un steak hâché frais de $100 \mathrm{~g}$ (cuisson $8 \mathrm{mn}$ ).

La dégradation de l'huile est modérée (tableau 6) et nettement moindre que celle décrite par certains auteurs [10].

Tous ces éléments concordent à montrer que l'huile de colza peut être utilisée à chaud comme elle l'est déjà dans de nombreux pays.

Il faut cependant prendre en compte l'aspect sensoriel. Si les produits frits paraissent bien acceptés, l'odeur dégagée spécialement lors des fritures peut être perçue comme désagréable par l'utilisateur.

Ainsi l'examen de la moyenne des scores obtenus sur une douzaine de tests d'odeur effectués dans l'année par notre panel montre que l'huile de colza est moins bien notée qu'une huile de tournesol à la première friture mais que l'écart s'atténue lors des fritures suivantes. Les défauts les plus marquants sont l'odeur âcre et piquante et parfois une odeur de poisson en particulier en cas de surchauffe (tableau 7).

\section{Conclusion}

D'après l'ensemble des résultats, rien n'empêche l'huile de colza d'être une huile multi-usages assaisonnement et cuisson.

Bien que riche en acides gras polyinsaturés, elle garde toute sa fraîcheur organoleptique si elle est conditionnée en emballage opaque et mieux encore si elle est conservée au froid.

Tableau 4.

\begin{tabular}{|c|c|c|c|c|c|c|c|c|c|c|}
\hline \multicolumn{11}{|c|}{ Colza : test de chauffages } \\
\hline & & \multicolumn{9}{|c|}{ Nombre de chauffages } \\
\hline & & $\mathbf{0}$ & 5 & 10 & 13 & 15 & 17 & 20 & 25 & 30 \\
\hline Acidité oléic & & 0,06 & 0,16 & 0,23 & 0,25 & 0,27 & 0,29 & 0,30 & 0,34 & 0,43 \\
\hline Transmissio & $0 \mathrm{~nm}(\%)$ & 70,6 & 24,3 & 11,1 & 6,4 & 4,2 & 2,4 & 1,3 & 0,5 & 0,2 \\
\hline Non chrom & aphié (\%) & 3,2 & 6,3 & 9,6 & 11,1 & 13,3 & 13,6 & 14,6 & 16,6 & 18,5 \\
\hline Composés | & (\%) & 3,5 & 12,8 & 19,3 & 23,8 & 26,3 & 30,9 & 33,1 & 37,2 & 42,8 \\
\hline \multirow{2}{*}{ C18:2 (\%) } & Cis & 16,95 & 15,68 & 14,49 & 13,84 & 13,15 & 12,93 & 12,49 & 11,67 & 10,94 \\
\hline & Trans & 0,13 & 0,14 & 0,11 & 0,12 & 0,11 & 0,12 & 0,13 & 0,13 & 0,14 \\
\hline \multirow[t]{2}{*}{ C18:3 (\%) } & Cis & 8,3 & 7,12 & 6,19 & 5,67 & 5,17 & 5,01 & 4,69 & 4,16 & 3,65 \\
\hline & Trans & 0,56 & 0,56 & 0,54 & 0,51 & 0,49 & 0,48 & 0,46 & 0,48 & 0,43 \\
\hline \multicolumn{2}{|c|}{ Polymères (\%) } & 0,3 & 7,7 & 12,3 & 15,3 & 17,5 & 19,9 & 22,3 & 26,4 & 28,7 \\
\hline \multicolumn{2}{|c|}{ Hauteur de mousse (mL) } & 4 & 4 & 15 & 32 & $>60$ & $>60$ & $>60$ & $>60$ & $>60$ \\
\hline \multicolumn{2}{|c|}{ Tocophérols (mg/100 g) } & 59,6 & 12,5 & 1,5 & & & & & & \\
\hline \multicolumn{11}{|c|}{ Colza : test de fritures } \\
\hline & & \multicolumn{9}{|c|}{ Nombre de fritures } \\
\hline & & $\mathbf{0}$ & 5 & 10 & 13 & 15 & 17 & 20 & 25 & 30 \\
\hline \multicolumn{2}{|c|}{ Acidité oléique (\%) } & 0,06 & 0,14 & 0,23 & 0,25 & 0,29 & 0,3 & 0,38 & 0,45 & 0,57 \\
\hline \multicolumn{2}{|c|}{ Transmission à 420 nm (\%) } & 70,6 & 6,2 & 1,4 & 0,7 & 0,4 & 0,3 & 0,2 & 0,1 & 0,1 \\
\hline \multicolumn{2}{|c|}{ Non chromatographié (\%) } & 3,2 & 5,2 & 6,5 & 8,4 & 8,8 & 9,7 & 10,5 & 13,2 & 13,8 \\
\hline \multicolumn{2}{|c|}{ Composés polaires (\%) } & 3,5 & 10 & 14,2 & 17,2 & 19,1 & 22,9 & 23,2 & 27,8 & 30,9 \\
\hline \multirow[t]{2}{*}{ C18:2 (\%) } & Cis & 16,95 & 16,08 & 15,56 & 14,91 & 14,64 & 14,36 & 14 & 13,15 & 12,64 \\
\hline & Trans & 0,13 & 0,10 & 0,10 & 0,14 & 0,12 & 0,15 & 0,11 & 0,13 & 0,13 \\
\hline \multirow[t]{2}{*}{ C18:3 (\%) } & Cis & 8,3 & 7,5 & 7,03 & 6,53 & 6,27 & 6,06 & 5,78 & 5,2 & 4,77 \\
\hline & Trans & 0,56 & 0,54 & 0,54 & 0,52 & 0,51 & 0,48 & 0,51 & 0,47 & 0,45 \\
\hline \multicolumn{2}{|c|}{ Polymères (\%) } & 0,3 & 5,2 & 7,5 & 10,3 & 11,1 & 12,9 & 14,6 & 17,3 & 20,8 \\
\hline \multicolumn{2}{|c|}{ Hauteur de mousse (ml) } & 4 & 4 & 4 & 9 & 11 & & 30 & 58 & $>60$ \\
\hline \multicolumn{2}{|c|}{ Tocophérols (mg/100 g) } & 59,6 & 14,2 & 3,7 & 1,8 & & & & & \\
\hline
\end{tabular}




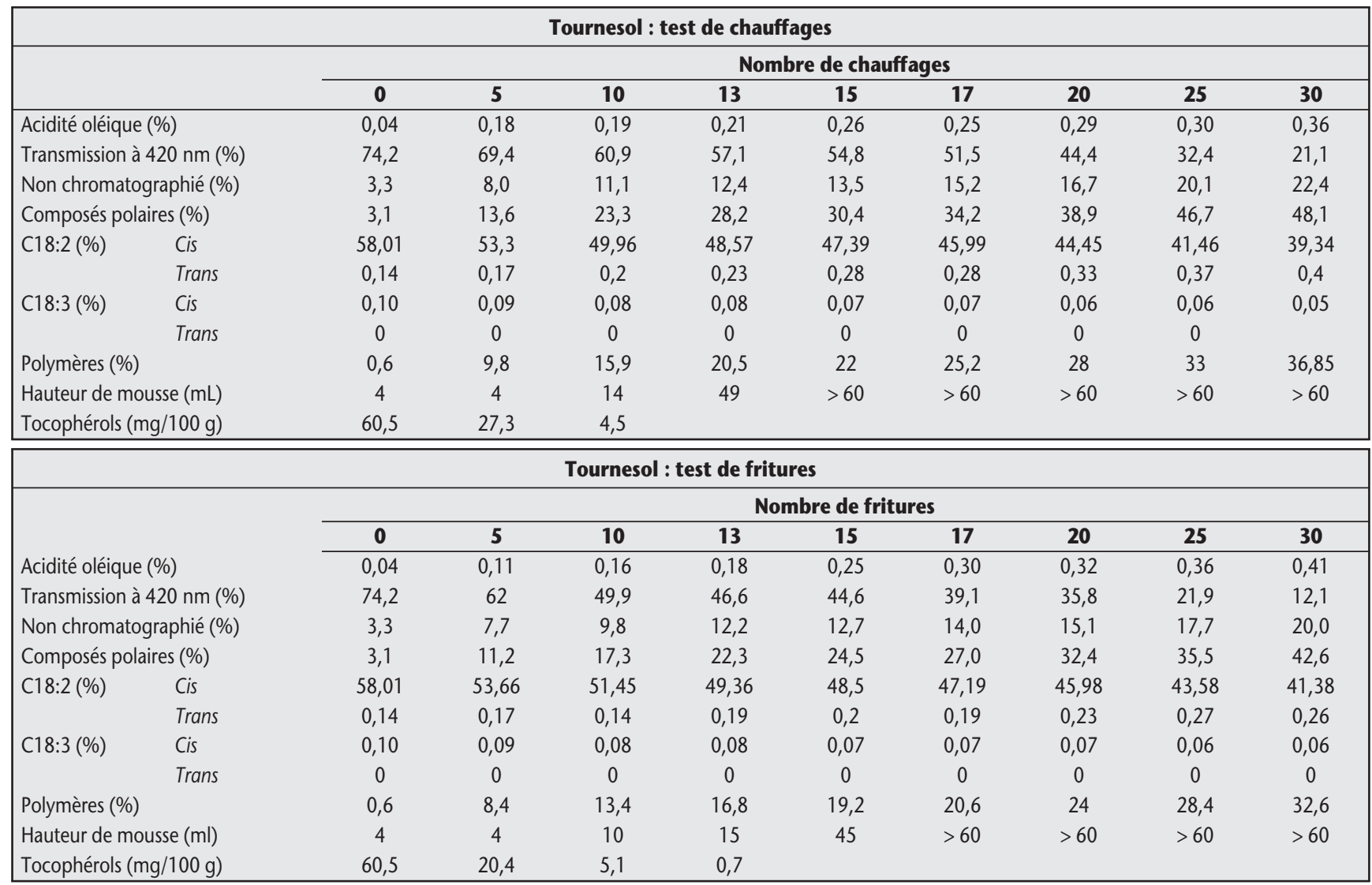

Tableau 6. Colza : cuisson plate.

\begin{tabular}{|lccc|}
\hline & Témoin & $\begin{array}{c}\text { Pommes de } \\
\text { terre rissolées }\end{array}$ & $\begin{array}{c}\text { Steak } \\
\text { hâché }\end{array}$ \\
\hline Acidité oléique (\%) & 0,05 & 0,09 & 0,09 \\
Composés polaires (\%) & 3,2 & 4,7 & 5,1 \\
Polymères (\%) & 0,2 & 0,55 & 1,1 \\
\hline
\end{tabular}

Tableau 7. Moyenne des scores au test d'odeur (échelle de 0 à 5).

\begin{tabular}{|lcc|}
\hline & Colza & Tournesol \\
\hline $1^{\text {re }}$ friture & 2,85 & 3,4 \\
$3^{\mathrm{e}}$ friture & 3,05 & 3,15 \\
$6^{\mathrm{e}}$ friture & 2,9 & 3,05 \\
\hline
\end{tabular}

L'encadrement réglementaire limitant à l'assaisonnement les huiles contenant plus de $2 \%$ d'acide linolénique ne paraît plus justifié [11]. En effet, la dégradation de l'huile de colza en usage à chaud n'est pas plus importante que celle d'autres huiles sans acide linolénique et reste en deçà de la limite de $25 \%$ de la teneur en composés polaires fixée par la réglementation française pour autant que des conditions raisonnables $\mathrm{d}^{\prime}$ utilisation soient conseillées par un étiquetage informatif $\left(\mathrm{T}^{\circ} \leq 180^{\circ} \mathrm{C}\right.$ Nombre maximum de fritures $\leq 10$ ).

L'huile de colza pourrait donc être utilisée pour des fritures profondes. Cependant, certains lui reprochent une odeur un peu forte et désagréable identifiée parfois à une odeur de poisson alors que ce n'est pas le cas de I'huile de tournesol pour laquelle les panélistes qualifient I'odeur comme odeur habituelle de friture.
Ce critère doit être pris en compte dans l'intérêt du consommateur. Pour cette raison, I'huile de colza peut être recommandée en usage assaisonnement et cuisson excluant ainsi la friture profonde.

\section{RÉFÉRENCES}

1. MARTIN A. Apports nutritionnels conseillés pour la population française. France : Tec et Doc, 2001 ; (CNERNA - CNRS - AFSSA, 2001).

2. AFSSA. Acides gras de la famille Omega 3 et système cardiovasculaire : intérêt nutritionnel et allégations. 2003 ; (Juillet).

3. COMBE N, BOUE-VAYSSE C. Apports alimentaires en acides linoléique et alpha-linolénique d'une population d'Aquitaine. OCL $2001 ; 8$ : 118-21.

4. COMBE N, BOUE-VAYSSE C. Face aux besoins et à la réalité des consommations, quelles sont les spécifications des différentes sources d'acides gras oméga 3 disponibles. OCL $2004 ; 11$ : 103-5.

5. HENON G, VIGNERON PY, et al. Rapeseed oil deodorization study using the responses surface methodology. Eur / Lipid Sci Technol 2001 ; 103 : 467-77.

6. VIGNERON PY, SPITCH P, AUDEGONDM. Huiles chauffées: étude des modifications chimiques au cours du chauffage. Revue Française des corps gras $1973 ; 8: 463-9$.

7. ZWOBADA F. Addition and edible oils in influence on quality. Revue Française des corps gras $1979 ; 11: 435-40$.

8. XIN-QUING XU, VIET HUNG TRAN, et al. Chemical and physical analyses and sensory evaluation of six deep-frying oils. J Am Oil Chem Soc $1999 ; 76$ : 1091-9.

9. NORMAND L, ESKIN NAM, PRZYBYLSKI R. Effect of tocopherols on the frying stability of regular and modified canola oils. J Am Oil Chem Soc 2001 ; 78 : 369-73.

10. KIATSRICHART S, BREWER M, et al. Pan-frying stability of Nusum Oil, a mid-oleic sunflower oil. J Am Oil Chem Soc $2003 ; 80: 479-83$.

11. REPUBLIQUE FRANCAISE. Decret RF No 73-139 du 12/02/73. 